\title{
Possibility Distribution of Wind Power Fluctuation
}

\author{
Bo Chen ${ }^{1}$ and Yuan $\mathrm{Li}^{2}$ \\ ${ }^{1}$ School of Electrical Engineering and Information, Sichuan University, Chengdu, \\ China \\ ${ }^{2}$ School of Electrical Engineering and Information, Sichuan University, Chengdu, \\ China \\ 1.cbhp1993@163.com,2yli@scu.edu.cn
}

\begin{abstract}
Volatility is the fixed property of wind power, how to describe the volatility of wind power quantitatively lacks effects methods currently. Based on the analysis of large quantities of testing data, it can be found that it is possible to adopt normal distribution to describe the possibility distribution of wind power fluctuating characteristics. Analysis demonstrates that min level component of wind power nearly occupies 2\%-5\% of the installed capacity of wind power station: when multiple wind power station outputs are overlapped, it can effectively reduce the proportion of min level component: the type of fans has very tiny effects on wind power fluctuation characteristics, but the current wind power out of wind power station has almost on influence on wind power fluctuation characteristics. Normal distribution also adapts to describe the possibility distribution of averaged power variation in adjacent time interval of wind power station, after the time interval is lengthened, due to decreased wind speed correlations, fluctuation characteristics of averaged power in adjacent intervals enhance.
\end{abstract}

Keywords: Wind power, wind power fluctuation, possibility density function, normal distribution

\section{Introduction}

With the gradually increase of the capacity of wind power connected into the power grid, people have paid more and more attention to the effects brought by the randomness of wind power. In fact, uncertainty and variation is fixed properties of power system operation. In recent hundred years, improving the infrastructure of power system and operation level itself is a process of coping with uncertainties and variations. The access of wind power aggravates the uncertainties and variations of power system, but it does not change these two essential properties of power system [1].

It usually adopts two methods to describe the randomness of wind power: One method is based on random sequence, literature [2-3] separately use Markov chain Monte Carlo and autoregressive integrated moving average model (ARIMA model) with amplitude limits to make wind power sequence modeling; the other is the method based on ARIMA probability density function (PDF). Existing literature makes research on distribution characteristics of wind speed [4], wind power efficiency [5-7], wind power unit current harmonics [8] and wind power prediction errors [9]. It is generally believed that wind speed satisfies Weibul1 distribution [4]. If the regulate wind speed meet the distribution of Weibul1, the distribution characteristic of wind power can be obtained through the relationship between wind speed and output power of wind turbine 
[8-9], but a large number of measured data show that the wind power are not satisfied with the common probability distribution. Literature [8] fitted the probability density function of different types of wind turbine generator system by adopting Weibull distribution and normal distribution. Literature [9] fitted the prediction error of wind power by adopting Beta distribution and then determines the size of the energy storage capacity with the prediction error of the wind power prediction. But the research on possibility distribution of wind power fluctuation lags behind significantly.

The data adopted in this paper is the 5s level output power of 20 stand-alone in a certain wind power plant in north China in August, 2012. the paper selected five sets of data for the research, of which adopted normal distribution, extreme distribution and generalized extreme value distribution, Logistic distribution and tocation-scale distribution to fit the probability distribution of $5 \mathrm{~s}$ level wind power $P_{i}^{5 s}\left(\mathrm{t}_{k}\right)$ and calculate the fitting index, thus set up the probability distribution model of wind power output. And then separate min component of wind power. At the same time use the min level data to set up the probability distribution model again. Analyzing the change of the accuracy of the data has an effect on output probability model of wind power plant.

\section{5s Level Data Probability Distribution of Wind Power Fluctuations}

To fitting the probability distribution of wind power, $P_{i}^{5 s}\left(\mathrm{t}_{k}\right)$ for the different probability distributions of the fitting, the defining fitting index is used to determine the fitting effect and select the optimum fitting distribution. Second, using $5 \mathrm{~s}$ level of first order difference $C_{i}^{5 s}\left(\mathrm{t}_{k}\right)=\mathrm{P}_{i}^{5 s}\left(\mathrm{t}_{k+1}\right)-\mathrm{P}_{i}^{5 s}\left(\mathrm{t}_{k}\right)$ to quantify the volatility of wind power and then study the probability distribution of sequence ${ }^{C_{i}^{5 s}\left(\mathrm{t}_{k}\right)}$, and calculate the fitting index I, select the optimum fitting distribution, then make a nonparametric and parameter hypothesis testing to the distribution.

\subsection{The Choice of 5s Level Wind Power Component Probability Density Function}

Label 1-5 on the five selected turbines. Because of the data range of wind power distribution are $(-\infty,+\infty)$, so the corresponding distribution are Normal distribution, Extreme value distribution, the Generalized Extreme value distribution, Logistic and $\mathrm{T}$ location-scale. Respectively fitting the above distribution, a fitting distribution diagram can be drawn in Figure 1; the fitting parameters are shown in Table 1.

Table 1. Parameter Estimation of Fitting Distribution

\begin{tabular}{|c|c|c|c|}
\hline Fitting distribution & Positional parameter mu & $\begin{array}{c}\text { Scale parameter } \\
\text { sigma }\end{array}$ & $\begin{array}{c}\text { Shape } \\
\text { parameter nv }\end{array}$ \\
\hline Normal & 321.624 & 381.832 & $\backslash$ \\
\hline Extreme value & 535.37 & 476.097 & $\backslash$ \\
\hline $\begin{array}{c}\text { Generalized } \\
\text { extreme value }\end{array}$ & 76.5435 & 120.686 & 0.978399 \\
\hline Logistic & 255.018 & 199.334 & $\backslash$ \\
\hline T location-scale & 156.868 & 179.882 & 1.65095 \\
\hline
\end{tabular}

Density 


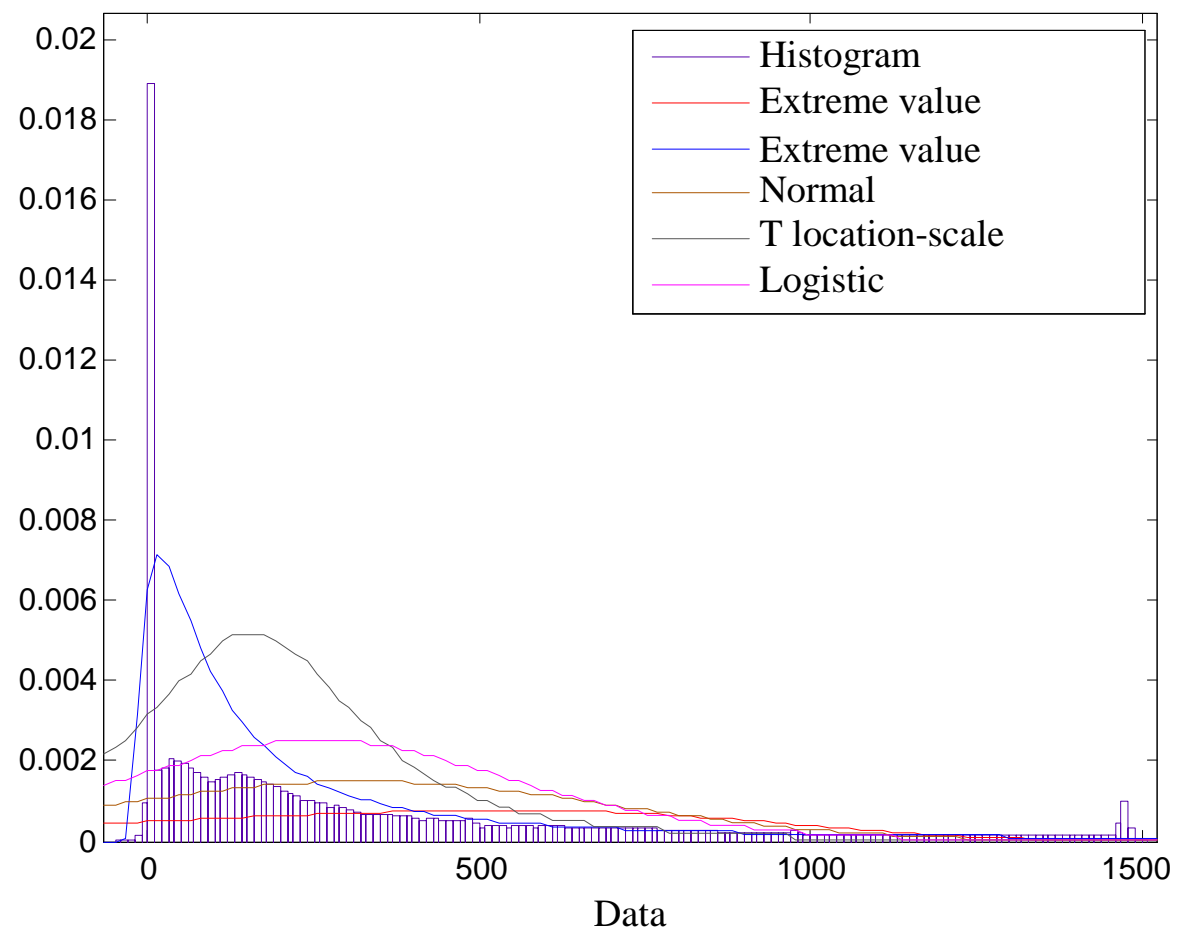

Figure 1. The Fitting of Distribution of $5 \mathrm{~s}$ Level Wind Power of $1 \mathrm{Fan}$

The aim of choosing different types of PDF probability density function is to make the fitting PDF as close to the frequency distribution histogram as possible. To quantitatively compare the fitting effect of the distribution function, define the fitting I:

$$
I=\sum_{\mathrm{i}=1}^{M}\left(y_{i}-\overline{N_{i}}\right)^{2}, y_{i}=f\left(\overline{C_{i}}\right)
$$

In this formula: $\mathrm{I}=1,2, \mathrm{M}, \mathrm{M}$ is the grouping number of the frequency distribution histogram, $\bar{N}_{i}$ and $\bar{C}_{\text {i }}$ respectively stands for the height and center position of NO. I straight square column, f stands for the fitting probability density function, $y_{i}=f\left(\overline{C_{i}}\right)$ stands for the corresponding value of probability density function located in the central position $\overline{C_{i}}$. The smaller I am, the more accurate the fitting obtained.

In order to calculate the fitting index I according to different PDF fitting probability distribution density function, we can draw the Table 2. Due to the fitting index of normal distribution is in a minimum, so the normal distribution fitted best to the $5 \mathrm{~s}$ level wind power of fan 1 in terms of the above probability distribution. 


\section{Table 2. The Fitting Index of Fan 1}

\begin{tabular}{|c|c|}
\hline Fitting distribution & Fitting index I \\
\hline Normal & 0.0340 \\
\hline Extreme value & 0.0362 \\
\hline Generalized extreme value & 0.0434 \\
\hline Logistic & 0.0371 \\
\hline T location-scale & 0.0383 \\
\hline
\end{tabular}

Combining with the practical considerations, due to the fan wind power is influenced by many factors and each factor cannot have absolute effect so the fitting result obey the normal distribution are reasonable.

Similarly we can fit and calculate the fitting index to the 5 s level wind power from 2 5 and obtain the optimum fitting distribution. The fitting distribution of fan 2 is similar to fan 1 , and the

Fitting distribution is as shown in Figure 2, the corresponding fitting index as shown in Table 3. due to the fitting index of normal distribution is in a minimum, the normal distribution fits best to the $5 \mathrm{~s}$ level wind power of fan 2 in terms of normal distribution.

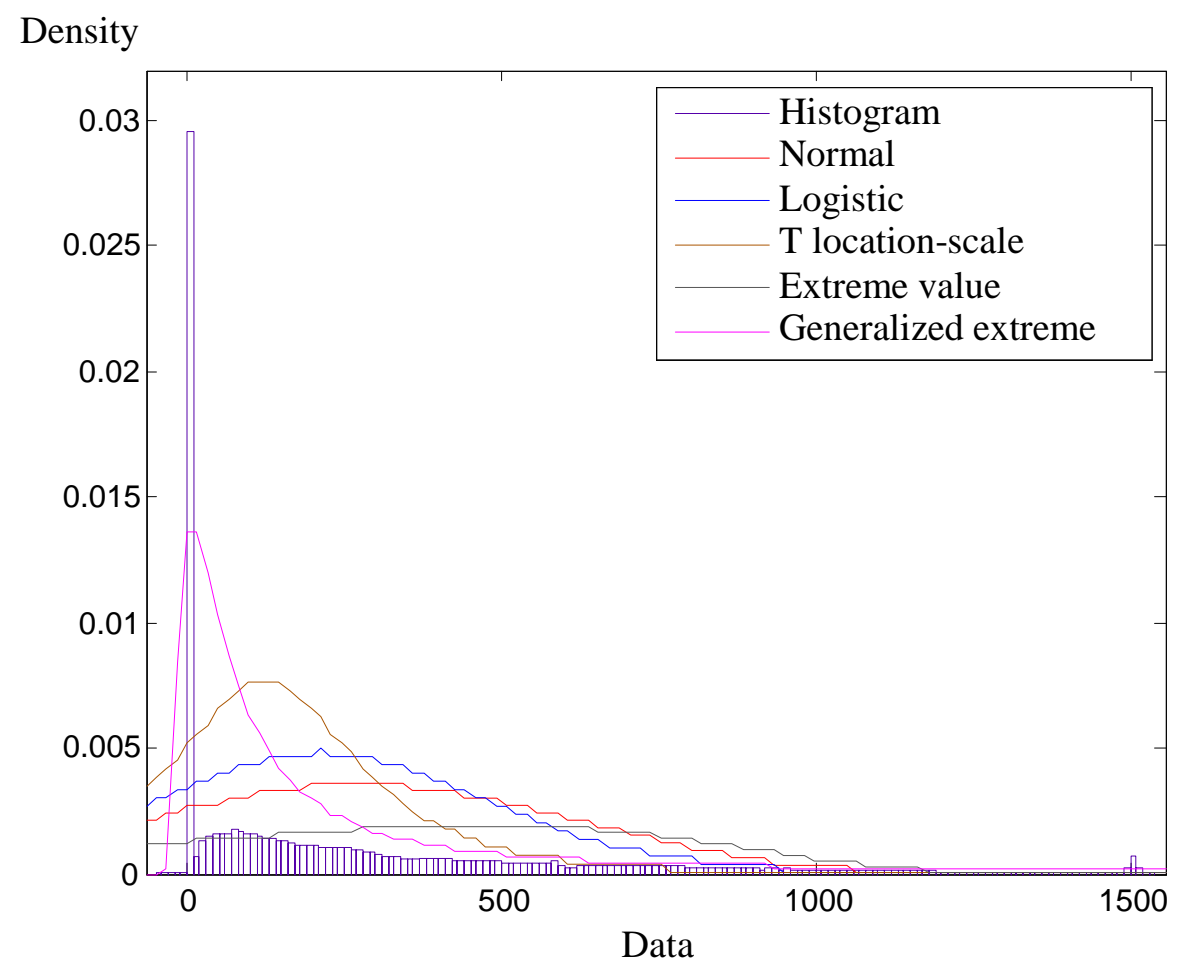

Figure 2. The Fitting of Distribution of 5s Level Wind Power of Fan 2 
Table 3. The Fitting Index of Fan 2

\begin{tabular}{|c|c|}
\hline Fitting distribution & Fitting index I \\
\hline Normal & 0.0939 \\
\hline Extreme value & 0.0945 \\
\hline Generalized extreme value & 0.1034 \\
\hline Logistic & 0.0956 \\
\hline T location-scale & 0.0968 \\
\hline
\end{tabular}

The fitting distribution of fan 3, 4, and 5 are similar in form or appearance, the corresponding fitting index is as shown in table 4. due to the extreme value distribution of fan 3, 4, 5 are in a minimum, the extreme value distribution fit best to the $5 \mathrm{~s}$ level wind power of fan 2, 3 and 4 in terms of the above probability distribution. Extreme value distribution is used to describe extreme events for a long time. Actually it is think that wind turbine generator system absorbed power from the power grid and long time without electricity is an extreme situation, so it is reasonable to obey the extreme value distribution.

Table 4. The Fitting Index from Fan 3-5

\begin{tabular}{|c|c|c|c|}
\hline \multirow{2}{*}{ Fitting distribution } & \multicolumn{3}{|c|}{ Fitting Index I } \\
\cline { 2 - 4 } & Fan 3 & Fan 4 & Fan 5 \\
\hline Normal & 0.1207 & 0.0803 & 0.0396 \\
\hline Extreme value & 0.1202 & 0.0797 & 0.0364 \\
\hline $\begin{array}{c}\text { Generalized } \\
\text { extreme value }\end{array}$ & 0.1277 & 0.0827 & 0.0419 \\
\hline Logistic & 0.1211 & 0.0806 & 0.0372 \\
\hline T location-scale & 0.1227 & 0.0816 & 0.0381 \\
\hline
\end{tabular}

\subsection{The First Difference Fitting of $5 \mathrm{~s}$ Level Wind Power}

$C_{i}^{5 s}\left(\mathrm{t}_{k}\right)$ Is the first difference of No. 1 fan of 5 s level wind power in ${ }^{t_{k}}$

$$
C_{i}^{5 s}\left(\mathrm{t}_{k}\right)=\mathrm{P}_{i}^{5 s}\left(\mathrm{t}_{k+1}\right)-\mathrm{P}_{i}^{5 s}\left(\mathrm{t}_{k}\right)
$$

Quantitative the wind power fluctuations of No. 1 fan in ${ }^{t_{k}}$, fitting the probability distribution of the wind power fluctuations (first difference).

Now we take fan 1 as an example to calculate the first difference of every moment. Because of the wind power value range is still, $(-\infty,+\infty)$ and symmetry to some degree. So the corresponding distribution is still Normal distribution, Extreme value distribution, the Generalized Extreme value distribution, and Logistic and t locationscale. Using the ditto fitting box in matlab to do a study on the probability distribution of the first difference of wind power and fitting respectively the above distribution, then the fitting of distribution parameters are shown in Table 5. 
Table 5. The Distribution Parameter Estimation of No. 1 Fan's First Difference

\begin{tabular}{|c|c|c|c|}
\hline Fitting distribution & $\begin{array}{c}\text { Positional } \\
\text { parameter mu }\end{array}$ & $\begin{array}{c}\text { Scale parameter } \\
\text { sigma }\end{array}$ & $\begin{array}{c}\text { Shape parameter } \\
\text { nv }\end{array}$ \\
\hline Normal & -0.000619235 & 30.5566 & $\backslash$ \\
\hline Extreme value & 15.7836 & 64.8091 & $\backslash$ \\
\hline $\begin{array}{c}\text { Generalized } \\
\text { extreme value }\end{array}$ & -12.63 & 74.9213 & -0.122489 \\
\hline Logistic & -0.343235 & 11.8125 & $\backslash$ \\
\hline T location-scale & -0.145992 & 5.05013 & 0.922759 \\
\hline
\end{tabular}

Adopting $x^{2}$ inspection method to conduct non-parametric hypothesis to the normal distribution fitting of fan 1 's first difference of wind power. If the inspection is unchecked, the inspection statistics as shown in Table 6 .

Table 6. Non-parametric Hypothesis Test Statistics of the Normal Distribution of Fan 1

\begin{tabular}{|c|c|}
\hline $\begin{array}{c}\mathrm{H} \text { (Take 0 or take } 1 \text { to accept the null } \\
\text { hypothesis) }\end{array}$ & 0 \\
\hline Chi-square statistic chi2stat & $5.2509 \mathrm{e}+04$ \\
\hline Degrees of freedom df & 0 \\
\hline The actual frequency O & {$[43250,471902]$} \\
\hline The theory of frequency E & {$[1.1083 \mathrm{e}+054.0432 \mathrm{e}+05]$} \\
\hline Boundary vector edges & {$[-1.4780 \mathrm{e}+03-24.1000599 .0000]$} \\
\hline
\end{tabular}

Parameter hypothesis test on the mean and standard deviation. In the case of unknown standard deviation, $t$ test is adapted to the mean value, and then test the variance by hypothesis test, the test statistics as shown in Table 7:

Table 7. The Parameter Testing Statistics of Fan 1

\begin{tabular}{|c|c|c|}
\hline & Mean value & Variance \\
\hline $\begin{array}{c}\text { h (Take 0 or take } 1 \text { to accept the } \\
\text { null hypothesis) }\end{array}$ & 0 & 0 \\
\hline P & 1.0000 & 0.9989 \\
\hline CI & {$[-0.0841,0.0828]$} & 1 \\
\hline tstat & $6.7810 \mathrm{e}-09$ & 162.9735768 .8910 \\
\hline df & 515151 & $\backslash$ \\
\hline sd & 30.5566 & 1 \\
\hline
\end{tabular}

So the fan 1 's first difference of wind power obey the normal distribution of $\mathrm{N}$ $(0.000619235,0.000619235)$

Similarly the probability distribution fitting of first order difference sequence from 2-5 are shown in figure 5. check its non-parametric hypothesis test and the parameter hypothesis test, all the text have been passed, the fitting parameters as shown in Table 8: 
Table 8. The first-order Differential Fitting Parameters from 1-5

\begin{tabular}{|c|c|c|}
\hline Wind turbines group & Mean value & The standard deviation \\
\hline No.1 & -0.000619235 & 30.5566 \\
\hline No.2 & -0.000978895 & 27.6753 \\
\hline No.3 & 0.00060346 & 27.6124 \\
\hline No.4 & $7.17946 \mathrm{e}-05$ & 31.6083 \\
\hline No.5 & -0.00276642 & 31.42 \\
\hline
\end{tabular}

\subsection{The Similarities and Differences Analysis of the Five Typhoons Electric Unit Probability Distribution}

Through the direct fitting of the probability distribution from 1-5, fan 1 and fan 2 respectively obey to the normal distribution of $\mathrm{N}(321.624,321.624)$ and $\mathrm{N}(272.906$, 272.906); fan 3, 4 and 5 respectively obey Extreme (505.265, 505.265), Extreme (574.721, 574.721), and Extreme (563.966, 563.966).

The first-order differential probability distribution from 1-5 all obey the normal distribution, from table 8 , you can see that the first-order differential probability distribution from 1-5 are similar, there little difference in the mean value and variance of the normal distribution. Consider that the difference of various fans' first order distribution is mainly due to difference space (location), the five fans we selected is continuous 1 to 5 , so the similar distribution is reasonable.

\section{Min Level Data Probability Distribution of Wind Power Fluctuations}

In the actual operation of the wind power plants, due to the limitation of data storage and management of the wind generator, all records power of second level data cannot be concentrated easily. We usually describe wind power fluctuations by the level of minutes and longer intervals. Extracted from 1 to 5 fan wind power data, calculate min level wind power $\mathrm{cm}$, and then make first order difference sequence analysis.

\subsection{The Algorithm Separation of Wind Power min Level Component}

Based on the min load of the algorithm in literature [10-12], the paper separate min level wind power by adopting the moving average method. imagine the length of sliding average time is $N_{\min }$, for the convenience of expression, $N$ as an even number, so the continuous component in the moment $t$, min can be calculated in the following form:

$$
\left\{\begin{array}{l}
P_{f t}=\left(P_{t-(N / 2-1)}+P_{t-(N / 2-2)}+\cdots+P_{t}+\cdots+P_{t+N / 2}\right) / N \\
P_{m t}=P_{t}-P_{f t} \\
t=N / 2, N / 2+1, \cdots, M-N / 2
\end{array}\right.
$$

In this formula: $P_{t}$ is measured in the first minute $t$ of the average power; $P_{f t}$ is the continuous component; $P_{m t}$ is the min level component, $P_{m t}$ is superimposed on the continuous component variation; $t$ is the measurement point; $M$ is the total number of points.

The choice of moving average length has certain randomness. If the length is too small, the wind power on the short wave will be reflected in the continuous component; if the length is too big, the trend of wind power change over time will be reflected in the min level 
component, the min level component is no longer a random variable. Literature [12] pointed out that the choice of moving average length of time depends on experience. For general characteristics of the load, sliding average time length selected 15 min relatively appropriate; To have a larger impact load (such as a large steel mills) control area, select a longer period of time (30 min) suitable length.

\subsection{The Choice of Wind Power min Stage Probability Density Function}

First extracted a min level wind power vector is from the $5 \mathrm{~s}$ level wind power vector, then calculating the first-order difference vector $\mathrm{cm}$ min level wind power. Next conduct fitting, parameter and non-parametric hypothesis test. Level 1 to 5 , fan min wind power order difference fitting situation as shown in Figure 3, the fitting parameters are shown in Table 9.

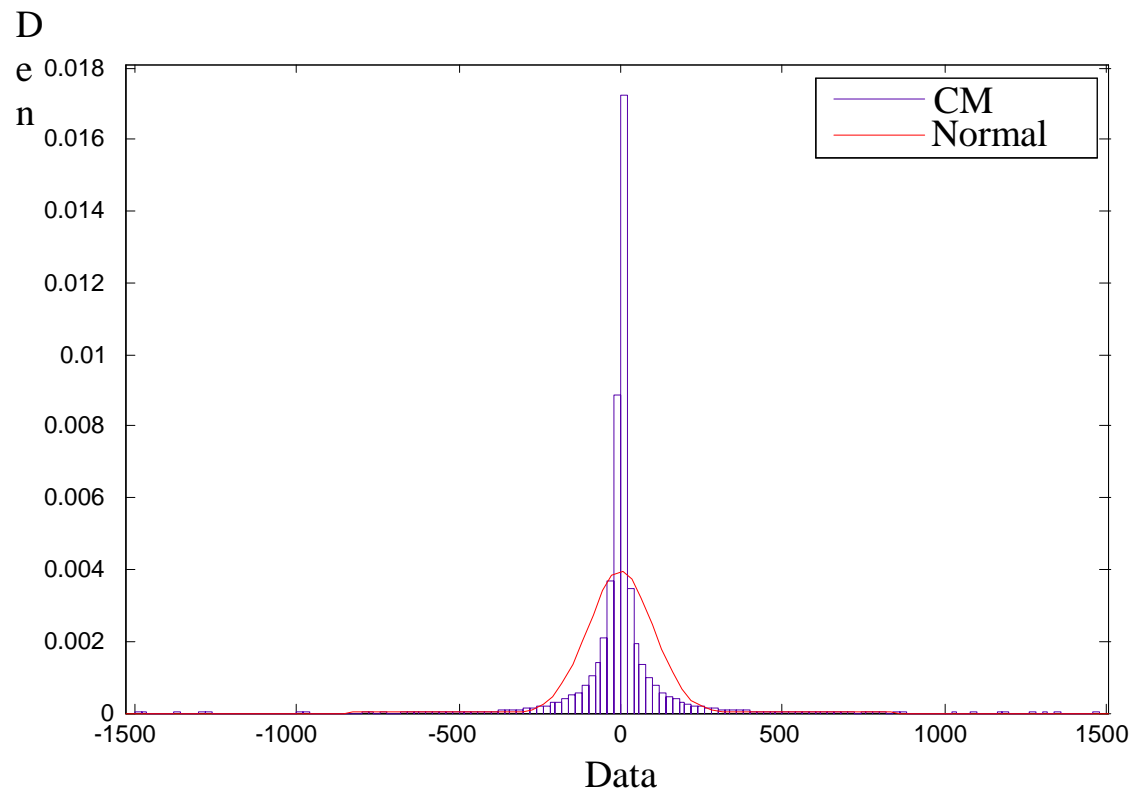

Figure 3. The Min Level Difference Fitting of Fan 1

Table 9. Min Level Difference Fitting Parameters from 1-5

\begin{tabular}{|c|c|c|}
\hline Wind turbines group & Mean value & The standard deviation \\
\hline 1 & 0.0226319 & 100.467 \\
\hline 2 & 0.019799 & 96.2354 \\
\hline 3 & 0.0151896 & 95.0712 \\
\hline 4 & 0.000768801 & 99.7006 \\
\hline 5 & 0.000652665 & 98.7367 \\
\hline
\end{tabular}

\section{Use $P_{i}^{m}\left(\mathrm{t}_{k}\right)$ to Replace $P_{i}^{P^{s s}\left(\mathrm{t}_{k}\right)}$ the Lost Information and its Measurement of Wind Power Fluctuations}

$5 \mathrm{~s}$ level wind power record 12 wind power per minute while min level only has one wind power value, which is bound to loss fluctuation information. In the above section, the fitting 
between $5 \mathrm{~s}$ level wind power from 1-5 and min level wind power fluctuation are the normal distribution, the fitting standard deviation parameters shown in Table 10.

Table 10. The Fitting Standard Deviation of $5 \mathrm{~s}$ Level and Min Level from 1-5

\begin{tabular}{|c|c|c|}
\hline \multirow{2}{*}{ Wind turbines group } & \multicolumn{2}{|c|}{ The standard deviation } \\
\cline { 2 - 3 } & sigma $^{5 s}(5 \mathrm{slevel})$ & sigm $^{\text {min }}(\mathrm{min}$ level $)$ \\
\hline No.1 & 30.5566 & 100.467 \\
\hline No.2 & 27.6753 & 96.2354 \\
\hline No.3 & 27.6124 & 95.0712 \\
\hline No.4 & 31.6083 & 99.7006 \\
\hline No.5 & 31.42 & 98.7367 \\
\hline
\end{tabular}

Standard deviation of normal distribution to a certain extent depict the aggregation degree of random variables near the average, the average figure 4 for 4 to 0 , the standard deviation of 1 to 4 of the normal distribution probability density function, from the figure we can see that the smaller the standard deviation, the greater the degree to the average aggregation and its fluctuation. By the table 11 shows no. 1 to 5 fan the $5 \mathrm{~s}$ level fitting standard deviations are far less than the min level fitting standard deviation. Although wind power fluctuation $P_{i}{ }^{m}\left(\mathrm{t}_{k}\right)$ are used to replace $P_{i}^{5 s}\left(\mathrm{t}_{k}\right)$ the lost part information, but the min level of volatility than $5 \mathrm{~s}$ level of volatility. Considering the actual situation, every $5 \mathrm{~s}$ wind power fluctuations on intuitive than wind power fluctuations is smaller per minute, and then $5 \mathrm{~s}$ level of first order differential fitting of the normal distribution of its standard deviation is smaller than the min level, which is in line with the actual situation.

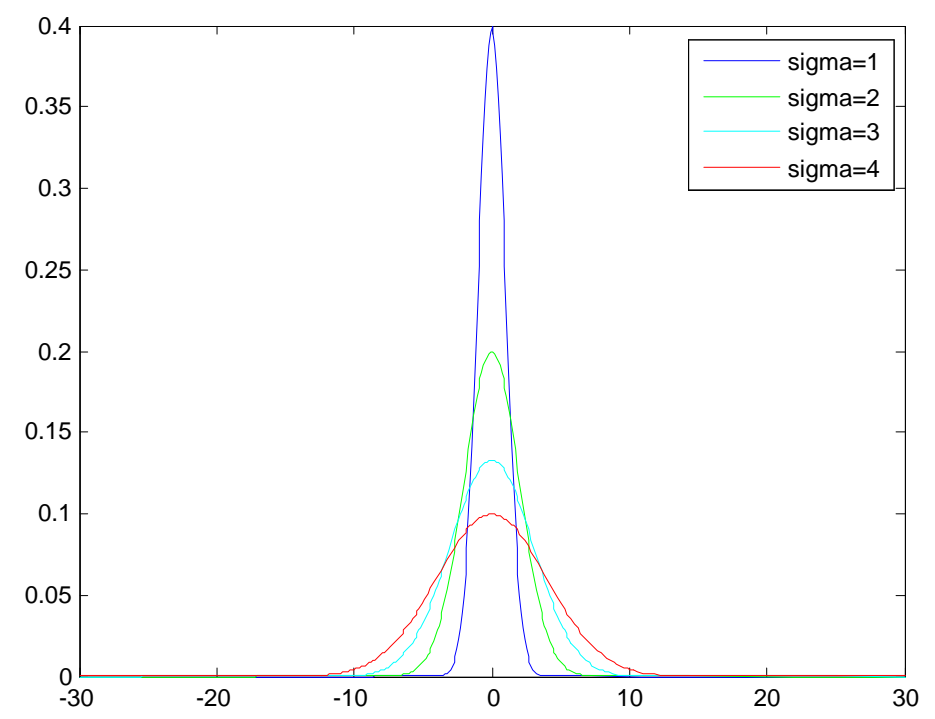

Figure 4. The Relationship between Normal Distribution and the Standard Deviation

$5 \mathrm{~s}$ wind power record 12 wind power per minute while min level only has one wind power value, of which lost a lot of volatility of information. Figure 5 stands for 10 minutes before the No. 1 fan $5 \mathrm{~s}$ level wind power and the min level wind power fluctuations, Figure 6 stands 
for ten minutes before the No. 1 fan $5 \mathrm{~s}$ level wind power and the min level wind power firstorder difference changes, its volatility loss information as shown in Figure 5, 6.

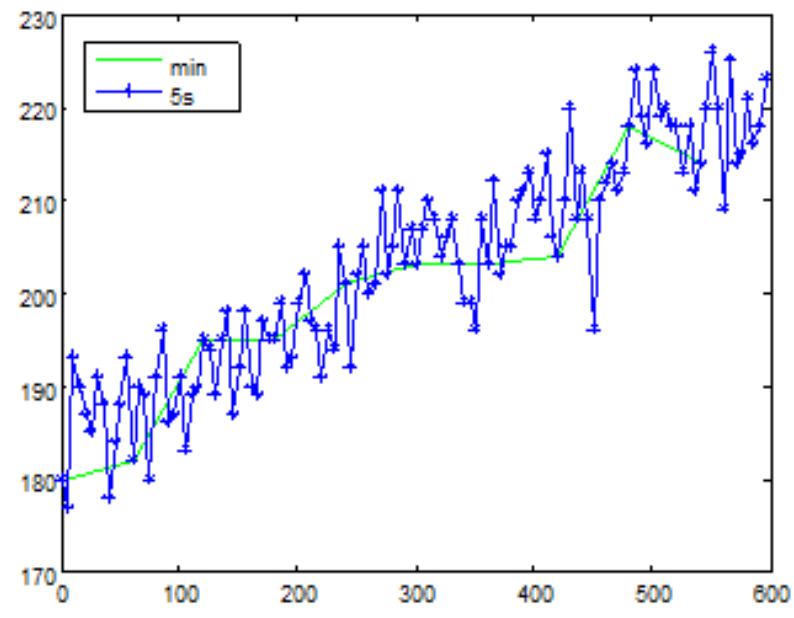

Figure 5. The 5s Level and Min Level wind Power in the Former 10 Minutes

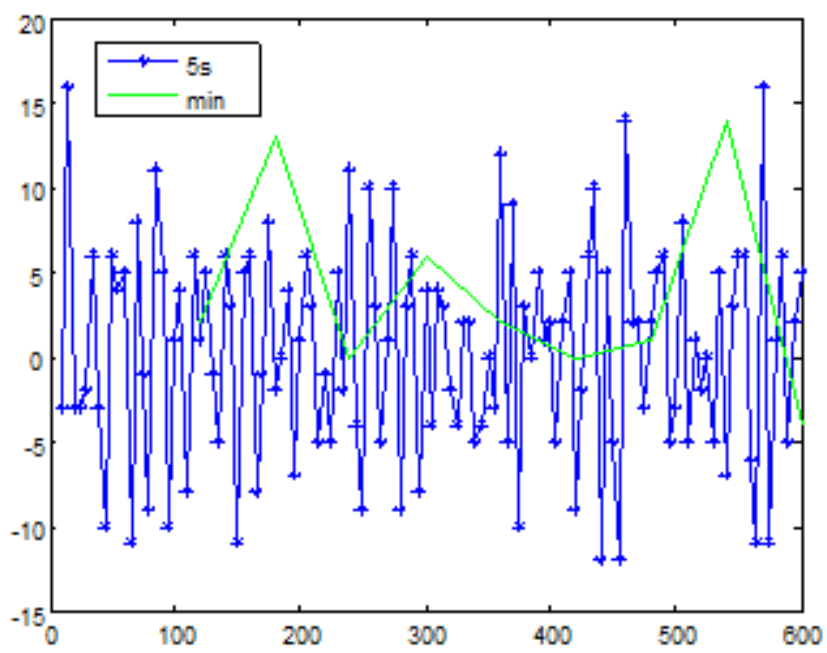

Figure 6. The 5s Level and Min Level Wind Differential in the Former 10 Minutes

$$
\operatorname{sig}=\left(1-\frac{\operatorname{sigma}^{5 s}}{\operatorname{sigma} a^{\min }}\right) \times 100 \% \text { is defined as damage index, } \operatorname{sigma}{ }^{\min } \text { quantitative }
$$

fluctuations type for the min level wind power in the first-order differential Gaussian fitting standard deviation, $\operatorname{sigma}^{5 s}$ a category $5 \mathrm{~s}$ wind power first order difference standard deviation of Gaussian fitting; Of 1 to 5 , fan sag index is calculated wave loss, we have the Table 11: 
Table 11. The Fluctuation Loss from Fan 1-5

\begin{tabular}{|c|c|}
\hline Wind turbines group & sig \\
\hline 1 & $69.585436 \%$ \\
\hline 2 & $71.2420793 \%$ \\
\hline 3 & $70.9560834 \%$ \\
\hline 4 & $68.2967806 \%$ \\
\hline 5 & $68.1779926 \%$ \\
\hline
\end{tabular}

From Table 12, we can see that $P_{i}^{m}\left(\mathrm{t}_{k}\right)$ substituted for $P_{i}^{5 s}\left(\mathrm{t}_{k}\right)$ wave loss is about $70 \%$. $P_{i}^{m}{ }^{m}\left(\mathrm{t}_{k}\right)$ Substituted for $P_{i}^{5 s}\left(\mathrm{t}_{k}\right)$ analysis of the volatile loss, fitting for the normal distribution of mean has very little change, but the standard deviation increases obviously as well as the wind power fluctuations.

In the summary, if expanding the time window width in the same unit to 1 minute, when studying partial wind power changes, despite that fluctuation is more obvious than that 5 minutes, the time window width of this 1-minute generates by accumulating 125 -minutes window widths. Therefore, it can only study the general fluctuation situation of this unit, or the situation of partial distortion will appear.

\section{Conclusion}

This paper adopts moving average method to extract min level component of the active power output of testing wind power. By analyzing actual measured data, it is found that it is possible to adopt normal distribution and extreme value distribution to describe $5 \mathrm{~s}$ level component of wind power quantitatively and use normal distribution to describe min level component of wind power quantitatively. For wind power station studied in this paper, normal distribution is more suitable than other distribution to describe fluctuating characteristics of wind power and wind energy, such as min level component of wind power and variations of wind power averaged values in adjacent intervals. Extreme distribution adopted in this paper can provide references for the analysis of the fluctuating characteristics of other wind power stations.

Analysis results demonstrate that active power of wind power station output involves smaller min level component. Taking the smooth averaged interval length of $30 \mathrm{~min}$ as the example, under $95 \%$ confidence coefficient, its min level component accounts for $2 \%-5 \%$ installed capacity of wind power station, and the percentage decreases with the increased of installed capacity of wind power station, min level component can be effectively after overlapping multiple wind power stations, after overlapping 5 wind power stations in this paper, its installed capacity will be $3652.5 \mathrm{Mw}$, under $95 \%$ confidence coefficient, min level component will be within $1.2 \%$.

The types of fans adopted in wind power station have no big influence on min level component, but the influence of the output of wind power station at some moment on min level component is almost zero. Normal distribution can not only be applied in the quantitative analysis of $5 \mathrm{~s}$ level and min level component effectively, but also be used to describe the possibility distribution of wind power averaged variation in adjacent intervals. In adjacent min, the averaged power variation of wind power is very small. Taking the example of the sum of 5 wind farm outputs, under $95 \%$ confidence level, the averaged power difference before and after $1 \mathrm{~min}$ is within $0.5 \%$ of installed capacity, after the further lengthening of time, time correlations of wind speed have been very weak, and the differences of wind power averaged values in adjacent intervals will increase gradually. 


\section{Reference}

[1] G. Papaefthymiou, IEEE Trans. on Energy Conversion, vol. 23, no. 1, (2008), p. 234.

[2] P. Chen, T. Pedersen and B. Bak-Jensen, IEEE Trans. on Power Systems, vol. 25, no. 2, (2010), p. 667.

[3] M. Ding, Y. Wu and L. Zhang, Proceedings of the CSEE, vol. 25, no. 10, (2005), p.107.

[4] J. Zhang, H. Cheng and L. Yao, Proceedings of the CSEE, vol. 29, no. 16, (2009), p. 1.

[5] D. Villanueva, J. L. Pazos and A. Fei, IEEE Trans. On Power Systems, vol. 26, no. 3, (2011), p. 1659.

[6] J. Usaola, "Electric Power Systems Research", vol. 80, no. 5, (2010), p. 528.

[7] S. Tentzerakis and S. Papathanassiou, IEEE Trans, On Energy Conversion, vol. 22,no. 1, (2007), p.150.

[8] A. Fabbri, T. Romdn and J. Abbad, IEEE Trans. on Power Systems, vol. 20, no. 3, (2005), p.1440.

[9] B. Kirb and, E. Hirst, IEEE Trans. on Power Systems, vol. 13, vol. 4, (1998), p. 1373.

[10] B. Kirby and E. Hirst, IEEE Trans. on Power Systems, vol. 13, no. 4, (1998), p.1379.

[11] B. Kirby and E. Hirst, Utilities Policy, vol. 8, no. 2, (1999), p. 75.

[12] A. Reigh, "Analysis of wind generation impact on ERCOT ancillary services requirements", New York: GE Energy, (2008).

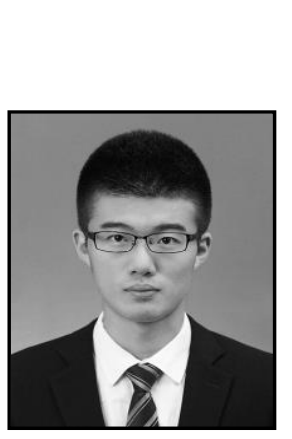

\section{Authors}

Bo Chen was born in Qinhuangdao, China in 1993. He will receive the B.Sc in electrical engineering in 2015 from the Sichuan University, where he is currently working as a research assistant.

His current research interests include control of electronic converters, photovoltaic energy systems and power quality.

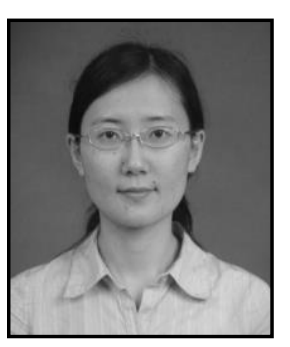

Yuan Li received the M.S. and Ph.D. degrees in electrical engineering from Wuhan University, Wuhan, China, in 2006 and 2009, respectively. From 2007 to 2009, she was a Visiting Scholar with the Department of Electrical and Computer Engineering, Michigan State University, East Lansing, where she was involved in research studies about renewable energy interface systems. She is currently a Lecturer with the College of Electrical Engineering and Information Technology, Sichuan University, Chengdu, China. Her research interests include Z-source inverters, photovoltaic inverters, power electronics applications in distributed generation, and smart grid. 